\title{
Higgs physics at the Future Circular Collider
}

\author{
David d'Enterria* \\ CERN, EP Department, 1211 Geneva, Switzerland \\ E-mail: dde@cern.ch
}

The unique Higgs physics opportunities accessible at the CERN Future Circular Collider (FCC) in electron-positron $(\sqrt{\mathrm{s}}=125,240,350 \mathrm{GeV})$ and proton-proton $(\sqrt{\mathrm{s}}=100 \mathrm{TeV})$ collisions, are succinctly summarized. Thanks to the large c.m. energies and enormous luminosities (plus clean experimental conditions in the $\mathrm{e}^{+} \mathrm{e}^{-}$case), many open fundamental aspects of the Higgs sector of the Standard Model (SM) can be experimentally studied:

(i) Measurement of the Higgs Yukawa couplings to the lightest fermions: u,d,s quarks (via rare exclusive $\mathrm{H} \rightarrow(\rho, \omega, \phi)+\gamma$ decays); and $\mathrm{e}^{ \pm}$(via resonant s-channel $\mathrm{e}^{+} \mathrm{e}^{-} \rightarrow \mathrm{H}$ production); as well as neutrinos (within low-scale seesaw mass generation scenarios).

(ii) Measurement of the Higgs potential (triple $\lambda_{3}$, and quartic $\lambda_{4}$ self-couplings), via double and triple Higgs boson production in pp collisions at $100 \mathrm{TeV}$.

(iii) Searches for new physics coupled to the scalar SM sector at scales $\Lambda_{\mathrm{NP}} \gtrsim 6 \mathrm{TeV}$, thanks to measurements of the Higgs boson couplings with subpercent uncertainties in $\mathrm{e}^{+} \mathrm{e}^{-} \rightarrow \mathrm{HZ}$.

(iv) Searches for dark matter in Higgs-portal interactions, via high-precision measurements of on-shell and off-shell Higgs boson invisible decays.

All these measurements are beyond the reach of pp collisions at the Large Hadron Collider. New higher-energy $\mathrm{e}^{+} \mathrm{e}^{-}$and pp colliders such as FCC are thus required to complete our understanding of the full set of SM Higgs parameters, as well as to search for new scalar-coupled physics in the multi-TeV regime.

38th International Conference on High Energy Physics

3-10 August 2016

Chicago, USA

${ }^{*}$ Speaker. 


\section{Introduction}

Despite its tremendous success describing many phenomena with high accuracy — crowned with the discovery of its last missing piece, the Higgs boson, in 2012 [1, 2] — many fundamental questions of the Standard Model (SM) of particle physics still remain open today. Our lack of understanding of the nature of dark matter, the origin of matter-antimatter asymmetry, the generation of neutrino masses, or how to tame the quadratically-divergent virtual SM corrections affecting the running of the Higgs boson mass between the widely separated electroweak and Planck scales ("fine tuning" problem), among others, are questions which likely will not be fully answered through the study of proton-proton (pp) collisions at the Large Hadron Collider (LHC). Searching for solutions to such fundamental problems, together with a complete experimental confirmation of the SM Higgs sector -including the unknown Yukawa couplings of the lightest fermions, as well as the triple $\lambda_{3}$ and quartic $\lambda_{4}$ Higgs self-couplings - requires both a new pp collider at higher center-of-mass (c.m.) energies, as well as a new high-precision $\mathrm{e}^{+} \mathrm{e}^{-}$machine with unprecedented luminosities to very accurately study the $\mathrm{H}$ boson properties. The Future Circular Collider (FCC) is a post-LHC project in a new 100-km tunnel under consideration at CERN [3], designed to deliver pp at $\sqrt{\mathrm{s}}=100 \mathrm{TeV}$ with $\mathcal{L}_{\text {int }}=0.2-2 \mathrm{ab}^{-1} / \mathrm{yr}$ integrated luminosities (FCC-hh) [4], as well as $\mathrm{e}^{+} \mathrm{e}^{-}$ over $\sqrt{\mathrm{s}}=90-350 \mathrm{GeV}$ with up to $80 \mathrm{ab}^{-1} / \mathrm{yr}$ (FCC-ee) [5]. Both machines are truly competitive "Higgs factories". Figure 1 shows the $\mathrm{H}$ boson production cross sections as a function of c.m.
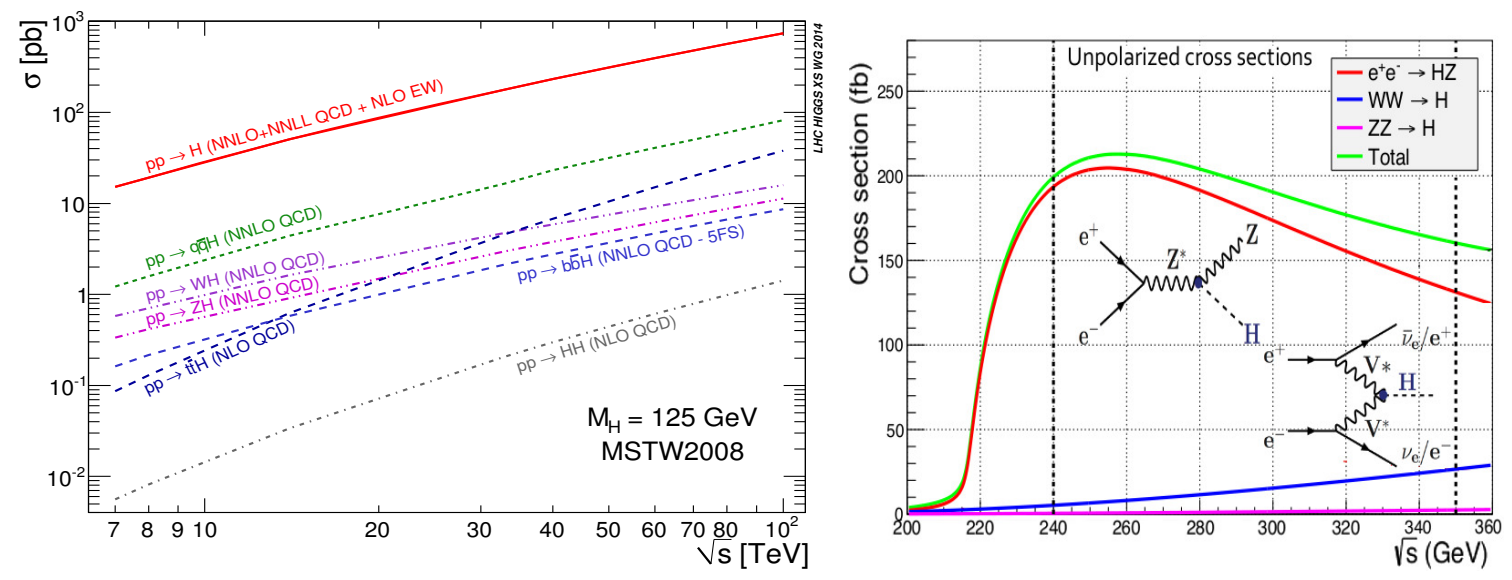

Figure 1: Higgs boson cross sections as a function of c.m. energy (total, and separated for different subprocesses) in pp (left) [6] and $\mathrm{e}^{+} \mathrm{e}^{-}$(right) [5] collisions.

energy at FCC-hh (left) and FCC-ee (right). Higgs production in DIS at FCC-eh, not discussed here, is also possible [7]. At the FCC-hh, gluon-gluon fusion ( $\mathrm{ggF}$ ) dominates the cross section, followed by vector-boson-fusion (VBF), and associated tit production. The sum of all contributions amounts to $\sigma(\mathrm{pp} \rightarrow \mathrm{H}+\mathrm{X}) \approx 0.9 \mathrm{nb}$ at $\sqrt{\mathrm{s}}=100 \mathrm{TeV}$ [6]. At the FCC-ee, the cross section (rates) peaks at $\sigma\left(\mathrm{e}^{+} \mathrm{e}^{-} \rightarrow \mathrm{H}+\mathrm{X}\right) \approx 200 \mathrm{fb}$ at $\sqrt{\mathrm{s}} \approx 240$ (250) GeV [5], dominated by Higgsstrahlung $\left(\mathrm{e}^{+} \mathrm{e}^{-} \rightarrow \mathrm{HZ}\right.$ ) with small VBF contributions ( $\mathrm{VV} \rightarrow \mathrm{H} \mathrm{e}^{+} \mathrm{e}^{-}, v v$ ). Both machines provide unparalleled opportunities to study the Higgs sector of the SM thanks to the enormous number of scalar bosons produced over $\sim 15$ and $\sim 20$ years of operation: up to $2 \cdot 10^{6}$ at FCC-ee with very low backgrounds and no pileup, and $2 \cdot 10^{10}$ at FCC-hh. Measurements of very precise Higgs couplings (with subpercent uncertainties), and of very rare and beyond the SM decays are thereby possible. 


\section{Generation of the lightest fermion (u, d, s; e; and v's) masses}

The SM Higgs boson couples to the fundamental fermions proportionally to their masses $\mathrm{m}_{\mathrm{f}}$, and thus its decays into the actual constituents of the stable visible matter in the Universe - formed by first generation fermions ( $\left.\mathrm{u} \overline{\mathrm{u}}, \mathrm{d} \overline{\mathrm{d}}, \mathrm{e}^{ \pm}\right)$with light masses $\mathcal{O}(0.5-10 \mathrm{MeV})$ - have extremely reduced branching ratios and cannot be directly measured at the LHC. The large and clean Higgs boson samples at FCC-ee will allow the measurements of very rare exclusive decays into light vectormesons $(\mathrm{VM})$ plus a photon $(\mathrm{H} \rightarrow \rho, \omega, \phi+\gamma$, with $\rho=(\mathrm{u} \overline{\mathrm{u}}-\mathrm{d} \overline{\mathrm{d}}) / \sqrt{2}, \omega=(\mathrm{uu}+\mathrm{d} \overline{\mathrm{d}}) / \sqrt{2}, \phi=\mathrm{s} \overline{\mathrm{s}})$ that are sensitive to the lightest quarks' Yukawas. The branching ratios for such processes are $\mathcal{O}\left(10^{-5}-10^{-6}\right)[6,8]$. The most promising one is $\mathrm{H} \rightarrow \rho(\pi \pi) \gamma$, with about 40 counts expected with low backgrounds. Determining the corresponding sensitivity to the $\mathrm{u} / \mathrm{d}$ quark Yukawa couplings requires dedicated studies given that the indirect $\mathrm{H} \rightarrow \gamma \gamma^{*} \rightarrow \mathrm{VM}+\gamma$ decays interfere with the direct $\mathrm{H} \rightarrow \mathrm{VM}+\gamma$ ones, and dilute the sensitivity to the latter. Of course, all these channels will be produced much more abundantly at FCC-hh, but the huge QCD (and pileup) backgrounds jeopardize a possible extraction of the corresponding u,d,s Yukawa couplings.

Measuring the electron Yukawa is even harder given the $\mathrm{e}^{ \pm}$lightness, and the only direct method to extract it is through resonant s-channel $\mathrm{e}^{+} \mathrm{e}^{-}$production running at the Higgs pole mass [9]. The resonant cross section for a $125-\mathrm{GeV}$ scalar of natural width $\Gamma_{\mathrm{H}}=4.1 \mathrm{MeV}$ is tiny, $\sigma\left(\mathrm{e}^{+} \mathrm{e}^{-} \rightarrow\right.$ $\mathrm{H})=1.64 \mathrm{fb}$. The actual cross section is further reduced accounting for the finite energy spread and initial state photon radiation (ISR) of the $\mathrm{e}^{ \pm}$beams. For a c.m. energy spread commensurate with the $\Gamma_{\mathrm{H}}$ natural width (dashed line in Fig. 2), reachable using monochromatization [11], the cross section becomes $\sigma\left(\mathrm{e}^{+} \mathrm{e}^{-} \rightarrow \mathrm{H}\right)=290$ ab [10]. Under these conditions, a preliminary study based on counting the number of events for

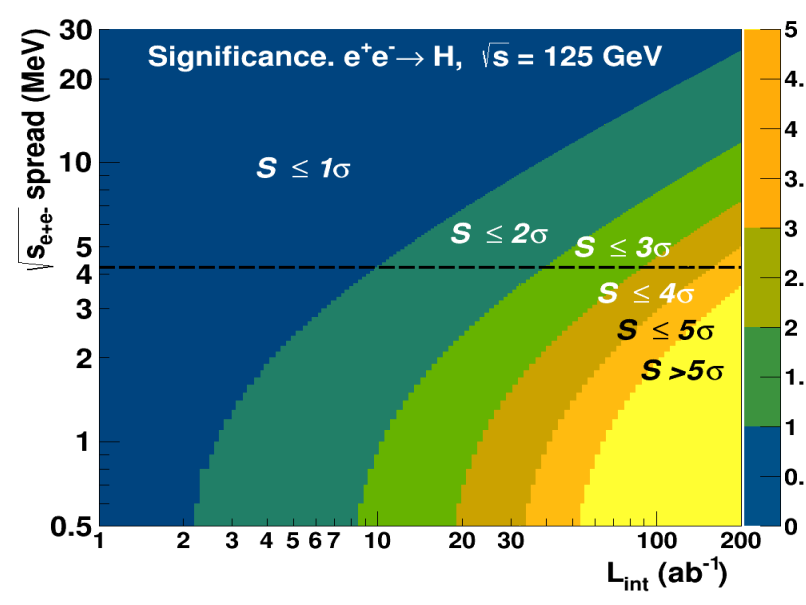

Figure 2: Significance contours for the $\mathrm{e}^{+} \mathrm{e}^{-} \rightarrow \mathrm{H}$ observation at $\sqrt{\mathrm{s}}=125 \mathrm{GeV}$ (combining 10 Higgs boson decays) in the $\sqrt{\mathrm{s}}$-spread vs. $\mathcal{L}_{\text {int }}$ plane at FCC-ee [9]. The dashed line shows the natural $\mathrm{H}$ boson width. signal and backgrounds in 10 different decay final-states in $\mathrm{e}^{+} \mathrm{e}^{-}$at $\sqrt{\mathrm{s}}=125.000 \pm 0.004 \mathrm{GeV}$, indicates that a $3 \sigma$ observation requires $\mathcal{L}_{\text {int }} \approx 90 \mathrm{ab}^{-1}$ (Fig. 2) [9]. For the target $\mathcal{L}_{\text {int }}=40 \mathrm{ab}^{-1} / \mathrm{yr}$ at $125 \mathrm{GeV}$, the significance of the signal is $2.1 \sigma$ which translates into limits on the $\mathrm{H} \rightarrow \mathrm{e}^{+} \mathrm{e}^{-}$branching ratio at $\times 1.2$ the SM expectation or, equivalently, a 95\% CL upper bound on $\times 1.1$ the SM prediction for the $\mathrm{e}^{ \pm}$Yukawa [9].

The generation of non-zero neutrino masses, called for by the observation of their flavor oscillations, is beyond the SM and requires new particles such as right-handed "sterile" $v$ 's. Phenomenologically-attractive scenarios have been considered [12] where sterile neutrinos $\mathrm{N}_{i}$ have masses around the electroweak scale, and thereby can be produced at FCC-ee and observed via $\mathrm{N}_{\mathrm{i}} \rightarrow \mathrm{H}+v$. Through the experimental study of mono-Higgs final states, FCC-ee has competitive sensitivities for $\mathrm{m}_{\mathrm{N}_{\mathrm{i}}} \approx 100-350 \mathrm{GeV}$ and values of the active-sterile mixing parameter down to $\left|\theta_{e}\right|^{2} \approx 10^{-5}$. 


\section{Determination of the Higgs potential (triple and quartic self-couplings)}

The Higgs sector of the SM cannot be considered to be fully confirmed experimentally until the strength of the Higgs boson to itself is measured. The SM Lagrangian parametrizes the Higgs self-interaction through its triple $\left(\lambda_{3}\right)$ and quartic $\left(\lambda_{4}\right)$ self-couplings, and their determination is crucial to confirm the shape of the Higgs potential and the mechanism of electroweak symmetry breaking [13]. Their direct determination is only possible through the production cross sections of two and three Higgs bosons. At the $\mathrm{LHC}(14 \mathrm{TeV})$ and $\mathrm{FCC}(100 \mathrm{TeV})$, the cross sections amount to $\sigma(\mathrm{HH}) \approx 0.05,1.9 \mathrm{pb}$ and $\sigma(\mathrm{HHH}) \approx 0.1,5 \mathrm{fb}$ [6]. However, different production subchannels contribute to the $\mathrm{HH}$ and $\mathrm{HHH}$ cross sections that do not directly involve $\mathrm{H}$ self-couplings, thereby diluting the final sensitivity on $\lambda_{3,4}$. At the end of the high-luminosity LHC running (HL-LHC, $14 \mathrm{TeV}, \mathcal{L}_{\text {int }}=3 \mathrm{ab}^{-1}$ ), the uncertainties on $\lambda_{3}$ will be of the order of $50 \%$ [14], whereas the measurement of $\lambda_{4}$ is out of reach.

The FCC-ee has a sensitivity to the $\lambda_{3}$ parameter comparable to that of the HL-LHC, through the high-precision study of the dominant $\mathrm{H}+\mathrm{Z}$ cross section which contains a small (energydependent) loop contribution involving the Higgs self-coupling [15]. However, a definite $\lambda_{3}$ measurement and constraints on $\lambda_{4}$, require a $100-\mathrm{TeV}$ pp collider such as FCC-hh. The precision achievable in the measurements of double and triple Higgs cross sections at FCC-hh, and associated $68 \%$ CL intervals on the $\lambda_{3}$ and $\lambda_{4}$ self-couplings are listed in Table 1 [6]. The trilinear self-

\begin{tabular}{l|c|c}
\hline \hline process & $\begin{array}{c}\text { (statistical) } \\
\text { precision on } \sigma_{S M}\end{array}$ & $\begin{array}{c}68 \% \text { CL interval on } \\
\text { Higgs self-couplings }\end{array}$ \\
\hline \hline$H H \rightarrow b \bar{b} \gamma \gamma$ & $3 \%$ & $\lambda_{3} \in[0.97,1.03]$ \\
\hline$H H \rightarrow b \bar{b} b \bar{b}$ & $5 \%$ & $\lambda_{3} \in[0.9,1.5]$ \\
\hline$H H \rightarrow b \bar{b} 4 \ell$ & $O(25 \%)$ & $\lambda_{3} \in[0.6,1.4]$ \\
$H H \rightarrow b \bar{b} \ell^{+} \ell^{-}$ & $O(15 \%)$ & $\lambda_{3} \in[0.8,1.2]$ \\
$H H \rightarrow b \bar{b} \ell^{+} \ell^{-} \gamma$ & - & - \\
\hline$H H H \rightarrow b \bar{b} b \bar{b} \gamma \gamma$ & $O(100 \%)$ & $\lambda_{4} \in[-4,+16]$ \\
\hline \hline
\end{tabular}

Table 1: Expected precision on SM cross sections for double and triple Higgs final-states reachable at FCC-hh (pp at $100 \mathrm{TeV}, 30 \mathrm{ab}^{-1}$ ), and associated $68 \% \mathrm{CL}$ ranges on $\lambda_{3}$ and $\lambda_{4}$ Higgs self-couplings. Details are provided in [6]. coupling can be measured with $3 \%$ uncertainties, whereas the quartic will be mildly constrained.

\section{Searches for new scalar-coupled physics}

With the Higgs boson discovered, the SM is now theoretically confronted to the hierarchy (aka. fine tuning or naturalness) problem, whereby quadratically-divergent SM virtual corrections affect the running of the Higgs boson mass between the widely separated electroweak and Planck scales. New particles are required to stabilize such untamed quantum corrections. Since the Higgs boson couples directly to any massive particle, the presence of any new physics has large chances to affect its couplings to the rest of SM particles. A powerful model-independent method to encode the effect of new physics from higher energies on experimental observables, is provided by the SM Effective Field Theory (EFT), which parametrizes possible new physics via a systematic expansion in a series of higher-dimensional operators composed of SM fields: $\mathcal{L}_{\text {eff }}=\mathcal{L}_{\mathrm{SM}}+\sum_{d=5}^{\infty} \frac{1}{\Lambda^{d-4}} \mathcal{L}_{d}$ with $\mathcal{L}_{d}=\sum_{i} c_{i}^{d} \mathcal{O}_{i}$, and unknown Wilson coefficients $c_{i}$ generated by decoupled new physics beyond the SM. Often, dim-6 operators $\mathcal{O}_{i}$ are the only ones considered (the Weinberg neutrino-mass is the 
unique dim-5 operator, and effects of $d>6$ operators are subleading in the decoupling assumption), i.e. $\mathcal{L}_{\text {eff }}=\mathcal{L}_{\mathrm{SM}}+\sum_{i} \frac{c_{i}}{\Lambda^{2}} \mathcal{O}_{i}$, where $\Lambda$ represents the scale of new interactions, and the coefficients $c_{i}$ depend on the details of its structure [16]. In the case of indirect (loop) constraints on new physics coupled to the Higgs boson, a useful back-of-the-envelope formula can be derived which relates $\Lambda$ to deviations of its couplings $\left(\delta g_{\mathrm{HXX}}\right)$ with respect to the expected SM values:

$$
\Lambda \gtrsim(1 \mathrm{TeV}) / \sqrt{\left(\delta \mathrm{g}_{\mathrm{HXx}} / \mathrm{g}_{\mathrm{HXx}}\right) / 5 \%} ;
$$

i.e. measurements of Higgs couplings with 5\% precision are sensitive to new physics at $\Lambda \gtrsim 1 \mathrm{TeV}$.

At lepton colliders, precise and model-independent Higgs measurements can be carried out using the recoil mass method in $\mathrm{e}^{+} \mathrm{e}^{-} \rightarrow \mathrm{HZ}$, which allows an accurate determination of the $\mathrm{H}$ boson 4-momentum irrespective of its decay mode, from the $\mathrm{Z} \rightarrow$ $\ell^{+} \ell^{-}(\ell=\mathrm{e}, \mu)$ decay reconstruction. At the FCC-ee, the highprecision $( \pm 0.4 \%)$ measurement of $\sigma_{\mathrm{e}^{+} \mathrm{e}^{-} \rightarrow \mathrm{HZ}} \propto g_{\mathrm{HZ}}^{2}$ provides a model-independent value of $g_{\mathrm{HZ}}$ to within $\pm 0.2 \%$. The total Higgs boson width $\Gamma_{\mathrm{H}}$ can also be obtained with $1 \%$ uncertainty combining the measured value of $\sigma_{\mathrm{e}^{+} \mathrm{e}^{-} \rightarrow \mathrm{H}(\mathrm{XX}) \mathrm{Z}} \propto$ $\Gamma_{\mathrm{H} \rightarrow \mathrm{XX}}$ with the known branching fractions, $\mathrm{BR}_{\mathrm{X}}=\Gamma_{\mathrm{H} \rightarrow \mathrm{XX}} / \Gamma_{\mathrm{H}}$, for different decays. The Higgs mass can be determined to within

\begin{tabular}{l|cc|cccc}
\hline \hline Parameter & $\begin{array}{c}\text { Current* } \\
7+8+13 \mathrm{TeV} \\
\left(\mathcal{O}\left(70 \mathrm{fb}^{-1}\right)\right.\end{array}$ & $\begin{array}{c}\text { HL-LHC }^{*} \\
14 \mathrm{TeV} \\
\left(3 \mathrm{ab}^{-1}\right)\end{array}$ & $\begin{array}{c}\text { FCC-ee } \\
\text { Baseline } \\
(10 \mathrm{yrs})\end{array}$ & $\begin{array}{c}\text { ILC } \\
\text { Lumi upgrade } \\
(20 \mathrm{yrs})\end{array}$ & $\begin{array}{c}\text { CEPC } \\
\text { Baseline } \\
(10 \mathrm{yrs})\end{array}$ & $\begin{array}{c}\text { CLIC } \\
\text { Baseline } \\
(15 \mathrm{yrs})\end{array}$ \\
\hline$\sigma(\mathrm{HZ})$ & - & - & $0.4 \%$ & $0.7 \%$ & $0.5 \%$ & $1.6 \%$ \\
$\mathrm{~g}_{\mathrm{ZZ}}$ & $10 \%$ & $2-4 \%$ & $0.15 \%$ & $0.3 \%$ & $0.25 \%$ & $0.8 \%$ \\
$\mathrm{~g}_{\mathrm{Ww}}$ & $11 \%$ & $2-5 \%$ & $0.2 \%$ & $0.4 \%$ & $1.6 \%$ & $0.9 \%$ \\
\hline $\mathrm{g}_{\mathrm{bb}}$ & $24 \%$ & $5-7 \%$ & $0.4 \%$ & $0.7 \%$ & $0.6 \%$ & $0.9 \%$ \\
$\mathrm{~g}_{\mathrm{cc}}$ & - & - & $0.7 \%$ & $1.2 \%$ & $2.3 \%$ & $1.9 \%$ \\
$\mathrm{~g}_{\tau \tau}$ & $15 \%$ & $5-8 \%$ & $0.5 \%$ & $0.9 \%$ & $1.4 \%$ & $1.4 \%$ \\
$\mathrm{~g}_{\mathrm{ti}}$ & $16 \%$ & $6-9 \%$ & $13 \%$ & $6.3 \%$ & - & $4.4 \%$ \\
$\mathrm{~g}_{\mu \mu}$ & - & $8 \%$ & $6.2 \%$ & $9.2 \%$ & $17 \%$ & $7.8 \%$ \\
$\mathrm{~g}_{\mathrm{e}^{+} \mathrm{e}^{-}}$ & - & - & $<100 \%$ & - & - & - \\
\hline $\mathrm{g}_{\mathrm{gg}}$ & - & $3-5 \%$ & $0.8 \%$ & $1.0 \%$ & $1.7 \%$ & $1.4 \%$ \\
$\mathrm{~g}_{\gamma \gamma}$ & $10 \%$ & $2-5 \%$ & $1.5 \%$ & $3.4 \%$ & $4.7 \%$ & $3.2 \%$ \\
$\mathrm{~g}_{\mathrm{Z} \gamma}$ & - & $10-12 \%$ & \multicolumn{5}{c}{ (to be determined) } & $9.1 \%$ \\
\hline$\Delta \mathrm{m}_{\mathrm{H}}$ & $200 \mathrm{MeV}$ & $50 \mathrm{MeV}$ & $11 \mathrm{MeV}$ & $15 \mathrm{MeV}$ & $5.9 \mathrm{MeV}$ & $32 \mathrm{MeV}$ \\
$\Gamma_{\mathrm{H}}$ & $<26 \mathrm{MeV}$ & $5-8 \%$ & $1.0 \%$ & $1.8 \%$ & $2.8 \%$ & $3.6 \%$ \\
$\Gamma_{\mathrm{inv}}$ & $<24 \%$ & $<6-8 \%$ & $<0.45 \%$ & $<0.29 \%$ & $<0.28 \%$ & $<0.97 \%$ \\
\hline \hline
\end{tabular}

Table 2: Summary of the best statistical precision attainable for Higgs observables at future $\mathrm{e}^{+} \mathrm{e}^{-}$colliders (FCCee [5], ILC [17], CEPC [18], CLIC [19]) compared to (modeldependent*) current LHC [20] and expected HL-LHC [14] pp results.

$\pm 11 \mathrm{MeV}$ from the measured recoil mass. Table 2 provides a summary of the best precision attainable for most Higgs boson properties at future $\mathrm{e}^{+} \mathrm{e}^{-}$machines (FCC-ee [5], ILC [17], CEPC [18], and CLIC [19]) compared to those today [20] and reachable at HL-LHC [14]. Lepton colliders provide a factor of at least 50 (10) improvement with respect to the present (HL-LHC) results that, in addition and at variance with the latter, do not depend on any SM fit. Among future $\mathrm{e}^{+} \mathrm{e}^{-}$colliders, FCC-ee typically features the highest precision thanks to its expected higher luminosities. Farther, the $2 \cdot 10^{10}$ scalars bosons produced at FCC-hh will also systematically improve the precision of all $\mathrm{H}$ couplings, preliminary studies $[21,6]$ indicate a potential precision of $1 \%$ for those with lower rates at $\mathrm{e}^{+} \mathrm{e}^{-}$machines: $g_{\mathrm{tt}}, g_{\mu \mu}$, and $g_{\mathrm{Z} \gamma}$.

The most precise coupling at FCC-ee $\left(\delta \mathrm{g}_{\mathrm{zz}} / \mathrm{g}_{\mathrm{zz}} \approx 0.15 \%\right)$ will allow setting limits on new scalar-coupled physics at $\Lambda \gtrsim 5.8 \mathrm{TeV}$ as per the simple estimate (4.1). Accurate theoretical analyses based on dim-6 EFT [16, 22] yield indeed $\Lambda \gtrsim 6 \mathrm{TeV}$ (Fig. 3, left). The same measurements can also be interpreted in terms of sensitivity to broad classes of SUSY models (such as the Constrained MSSM, or natural SUSY) effectively covering phase space corners beyond the LHC reach [23, 24]. 


\section{Searches for Higgs-portal dark matter (DM)}

The SM describes only $4 \%$ of the universe energy budget, the rest being in the form of unknown DM (and dark energy) contributions, pointing to the existence of new massive particles (such as e.g. SUSY partners, heavy $v$ 's, axions,...). In Higgs-portal models [25], the $\mathrm{H}$ boson acts as a mediator between the SM and DM particles, playing a central role in the evolution of the early universe. Attractive scenarios exist for DM candidates $(\phi)$ lighter than $m_{\mathrm{H}, \mathrm{Z}} / 2$, consistent with the measured DM thermal relic abundance in the universe, with DM freezing out through resonant $\mathrm{H}$ (or $\mathrm{Z}$ ) exchanges. In such cases, the measurements of the invisible $\mathrm{H}$ and $\mathrm{Z}$ widths provide the best collider options to test such scenarios [26]. Current invisible $\mathrm{H}$ decays limits are $\mathrm{BR}(\mathrm{H} \rightarrow$ inv. $)<0.24(95 \% \mathrm{CL})$ at the $\mathrm{LHC}[27]$, and are expected to reach $\mathrm{BR}(\mathrm{H} \rightarrow$ inv. $)<0.06$ at HL-LHC (Table 2). At the FCC-ee, the $\mathrm{HZ}\left(\ell^{+} \ell^{-}\right)$final state can be used to directly measure $\Gamma_{\text {inv }}$ (a $5 \sigma$ observation is possible down to $\mathrm{BR}=1.7 \pm 0.1 \%$ ) [28], in events where its decay products escape undetected. If unobserved, a $0.5 \%$ upper limit $(95 \% \mathrm{CL})$ [5] can be set on this branching ratio (Fig. 3, right), placing DM bounds a factor of 50 (10) better than those at LHC (HL-LHC), and being also competitive with the reach of planned direct detection experiments for $\mathrm{m}_{\phi}<10 \mathrm{GeV}$ [28].
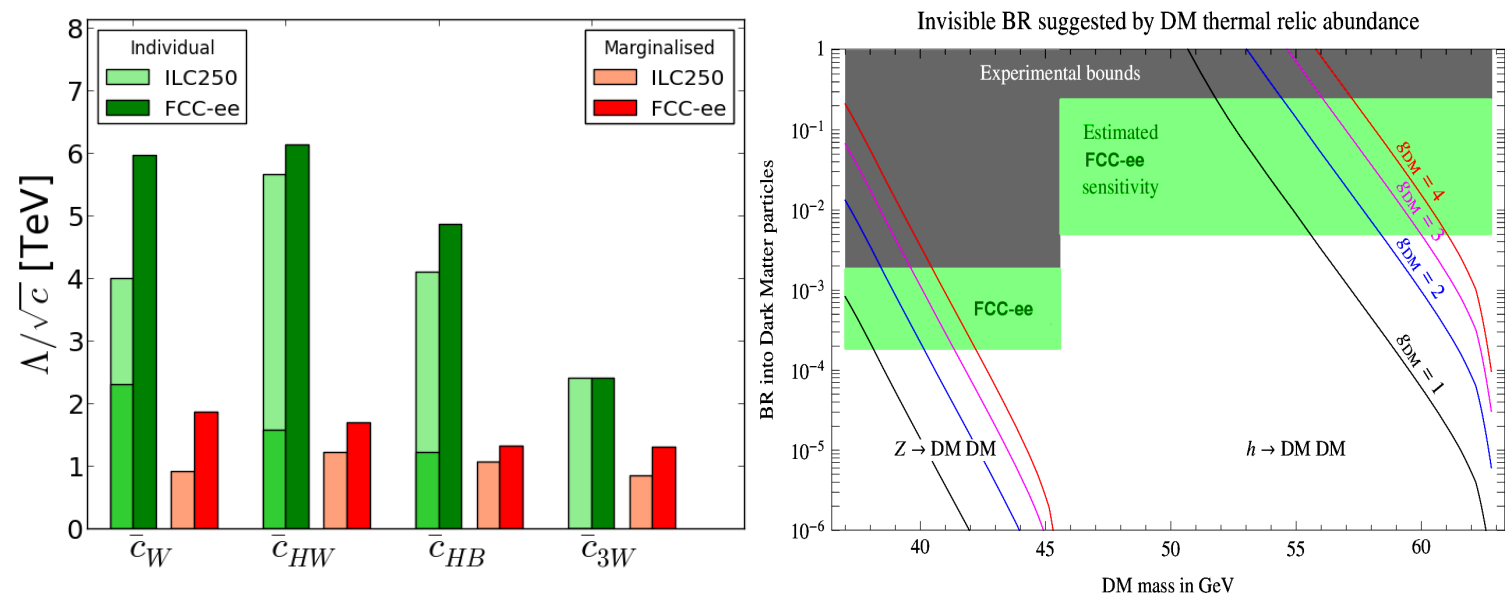

Figure 3: Left: Sensitivity reach to new physics scales $\left(\Lambda / \sqrt{c_{i}}\right)$, encoded in four dim-6 operator $c_{i}$ coefficients, of precision Higgs (and triple gauge boson couplings) measurements at FCC-ee and ILC [16]. Right: FCC-ee sensitivity for rare $\mathrm{H}$ (and Z) decays into DM pairs in the $\mathrm{BR}_{\mathrm{H}, \mathrm{Z} \rightarrow \phi \phi}$ vs. $\mathrm{m}_{\phi}$ plane [26].

For DM particles heavier than the Higgs boson, off-shell $\mathrm{H}$ decays into DM can be searched in pp events characterized by missing energy (from the $\mathrm{H}^{\star} \rightarrow \phi \phi$ decay) accompanied by extra particle production (gluon ISR in ggF, forward-backward jets in VBF, associated $\mathrm{t} \overline{\mathrm{t}}, \ldots$ ) as done at the LHC [20]. Theoretical studies indicate that the FCC-hh can place strong constraints on Higgsportal couplings $\left|c_{\phi}\right| \approx 0-3.5$ for scalar DM masses $m_{\phi}=150-500 \mathrm{GeV}$ (see details in [6, 29]).

\section{Summary}

The Future Circular Collider (FCC) will provide unparalleled luminosities $\mathcal{O}\left(1-20 \mathrm{ab}^{-1}\right) / \mathrm{yr}$ in pp $(\sqrt{\mathrm{s}}=100 \mathrm{TeV})$ and $\mathrm{e}^{+} \mathrm{e}^{-}(\sqrt{\mathrm{s}}=125-350 \mathrm{GeV})$ collisions, totalling $2 \cdot 10^{10}$ and $2 \cdot 10^{6}$ Higgs bosons produced over their respective expected operation times, and opening up measurements with 
$\mathcal{O}(50)(\mathcal{O}(10))$ times better precision than those reachable at LHC (HL-LHC). The unique FCC Higgs physics opportunities include fully closing the SM scalar sector (measuring the unknown Yukawas of the first-generation fermions, as well as the triple and quartic Higgs self-couplings), and discovering (or placing bounds on) scalar-coupled new physics well into the multi- $\mathrm{TeV}$ regime.

\section{References}

[1] G. Aad et al. [ATLAS Collaboration], Phys. Lett. B 716 (2012) 1

[2] S. Chatrchyan et al. [CMS Collaboration], Phys. Lett. B 716 (2012) 30

[3] M. Benedikt, B. Goddard, D. Schulte, F. Zimmermann and M. J. Syphers, IPAC-2015-TUPTY062

[4] F. Zimmermann, M. Benedikt, X. Buffat and D. Schulte, Proceeds. JACoW-IPAC2016-TUPMW037

[5] M. Bicer et al. [TLEP Design Study Working Group], JHEP 1401 (2014) 164; D. d'Enterria, Proceeds. 17th Lomonosov Conf., Moscow, Aug. 2015; arXiv:1602.05043 [hep-ex].

[6] R. Contino et al., arXiv:1606.09408 [hep-ph]

[7] M. Klein, Annalen Phys. 528 (2016) 138

[8] G. Perez, Y. Soreq, E. Stamou and K. Tobioka, Phys. Rev. D 93 (2016) 013001

[9] D. d'Enterria, G. Wojcik, R. Aleksan, 7th, 8th, 10th FCC-ee Physics Workshops (Geneva, June 2014; LPNHE-Paris, Oct. 2014; CERN, Feb. 2016); and in preparation

[10] S. Jadach and R. A. Kycia, Phys. Lett. B 755 (2016) 58

[11] M. A. Valdivia, A. Faus-Golfe and F. Zimmermann, Proceeds. JACoW-IPAC2016-WEPMW009

[12] S. Antusch, E. Cazzato and O. Fischer, arXiv:1612.02728 [hep-ph]

[13] A. Djouadi, Phys. Rept. 457 (2008) 1

[14] S. Dawson et al., arXiv:1310.8361 [hep-ex]

[15] M. McCullough, Phys. Rev. D 90 (2014), 015001 [Erratum-ibid. 92 (2015), 039903]

[16] J. Ellis and T. You, JHEP 1603 (2016) 089

[17] K. Fujii et al. [ILC Collaboration], arXiv:1506.05992 [hep-ph]

[18] M. Ahmad et al. [CEPC-SPPC Study Group], IHEP-CEPC-DR-2015-01, IHEP-TH-2015-01 (2015)

[19] H. Abramowicz et al. [CLIC Collaboration], arXiv:1608.07538 [hep-ex]

[20] G. Aad et al. [ATLAS and CMS Collaborations], JHEP 1608 (2016) 045

[21] M. L. Mangano, T. Plehn, P. Reimitz, T. Schell and H. S. Shao, J. Phys. G 43 (2016), 035001

[22] J. de Blas et al., JHEP 1612 (2016) 135

[23] O. Buchmueller, M. Citron, J. Ellis, et al., Eur. Phys. J. C 75 (2015) 469

[24] J. Fan, M. Reece and L. T. Wang, JHEP 1508 (2015) 152

[25] A. Djouadi, A. Falkowski, Y. Mambrini and J. Quevillon, Eur. Phys. J. C 73 (2013) 2455

[26] A. De Simone, G. F. Giudice and A. Strumia, JHEP 1406 (2014) 081; A. Strumia, 9th FCC-ee Physics Workshop, SNS-Pisa, Feb. 2015, http://www.sns. it/eventi/9th-fcc-ee-physics-workshop

[27] V. Khachatryan et al. [CMS Collaboration], arXiv:1610.09218 [hep-ex]

[28] O. Cerri, M. de Gruttola, M. Pierini, A. Podo and G. Rolandi, arXiv:1605.00100 [hep-ex]

[29] N. Craig, H. K. Lou, M. McCullough and A. Thalapillil, JHEP 1602 (2016) 127 